\title{
Labiobaetis from the Kingdom of Saudi Arabia (Insecta, Ephemeroptera, Baetidae)
}

\author{
Jean-Luc Gattolliat ${ }^{1,2}$, Boris C. Kondratieff ${ }^{3}$, \\ Thomas Kaltenbach ${ }^{1,2}$, Hathal M. Al Dhafer ${ }^{4}$
}

I Museum of Zoology, Palais de Rumine, Place de la Riponne 6, 1014 Lausanne, Switzerland 2 Department of Ecology and Evolution, Biophore, University of Lausanne, 1015 Lausanne, Switzerland 3 Department of Bioagricultural Sciences and Pest Management, Colorado State University, Fort Collins, CO 80523, USA 4 Plant Protection Department, College of Food and Agriculture Sciences, King Saud University, Riyadh 11451, P. O. Box 2460, Saudi Arabia

Corresponding author: Jean-Luc Gattolliat (jean-luc.gattolliat@vd.ch)

Academic editor: B. Price | Received 26 March 2018 | Accepted 25 May 2018 | Published 16 July 2018

http://zoobank.org/D06A30D9-98C4-4B47-912F-F8A318EC59A9

Citation: Gattolliat J-L, Kondratieff BC, Kaltenbach T, Al Dhafer MM (2018) Labiobaetis from the Kingdom of Saudi Arabia (Insecta, Ephemeroptera, Baetidae). ZooKeys 774: 77-104. https://doi.org/10.3897/zookeys.774.25273

\begin{abstract}
Mayfly larvae and imagos were collected at approximately 50 localities of the Kingdom of Saudi Arabia (KSA). Included in this material, three species of Labiobaetis Novikova \& Kluge, 1987 are recorded, two of them being new to science. Labiobaetis potamoticus Gattolliat \& Al Dhafer, sp. n. is described from both larvae and adults, whereas Labiobaetis alahmadii Gattolliat \& Al Dhafer, sp. n. is only known from the larval stage. The two species are compared morphologically with Palearctic and Afrotropical species of Labiobaetis. A third species, Labiobaetis glaucus (Agnew, 1961) is reported for the first time from the Arabian Peninsula. The species was originally described from South Africa and subsequently reported from the east and northeast of Africa. A molecular reconstruction including 18 Afrotropical and Palearctic species of Labiobaetis was performed using $658 \mathrm{bp}$ of the mitochondrial gene CO1. The reconstruction highly supported the validity of the two new species and confirmed the occurrence of L. glaucus in KSA.
\end{abstract}

\section{Keywords}

Arabian Peninsula, mayflies, new species, new records

Copyright Jean-Luc Gattolliat et al. This is an open access article distributed under the terms of the Creative Commons Attribution License (CC BY 4.0), which permits unrestricted use, distribution, and reproduction in any medium, provided the original author and source are credited. 


\section{Introduction}

Labiobaetis Novikova \& Kluge, 1987 is a species-rich genus with an almost worldwide distribution (only absent in Neotropical Region); it is mainly diversified in Afrotropical (28 species) and Oriental realms (23 species) (Gattolliat and Nieto 2009). The status and validity of the genus has often been the subject of controversy during the last two decades (Fujitani 2008; Fujitani et al. 2003; Gattolliat 2001; Gattolliat and Staniczek 2011; Kluge 2012; Kluge and Novikova 2016; Kubendran et al. 2015; LugoOrtiz and McCafferty 1997; Lugo-Ortiz et al. 1999). Molecular reconstructions indicated that the concept of Labiobaetis is most probably at least diphyletic (Gattolliat et al. 2008; Monaghan et al. 2005). Larvae of Labiobaetis generally colonize the more lentic portion of streams and rivers with rich aquatic vegetation where it can be the most abundant mayfly.

The distribution of the genus in Arabian Peninsula seems restricted to the southwestern Kingdom of Saudi Arabia (KSA) and Yemen despite potential suitable habitats present in Oman, Jordan and the United Arab Emirates (Gattolliat and Sartori 2008; Gattolliat et al. 2012).

Relatively little information is available on the freshwater habitats and faunas of the Arabian Peninsula and, in particular, of the KSA. The vast landscape of KSA is one of the driest and hottest countries in the world and has almost no runoff, surface water or perennial rivers (Alshareef 1995, Alkolibi 2002, Al-Rashed and Sherif 2000). Other than the Midwestern Region and the southwestern mountains, the average annual precipitation in KSA ranges from $80 \mathrm{~mm}$ to $140 \mathrm{~mm}$ and maximum summer temperatures often exceeds $45^{\circ} \mathrm{C}$ (Alkolibi 2002). Seasonal rainfall occurs more frequently and in greater quantity in the southwestern Hejaz and Asir mountains, therefore most permanent and semi-permanent lotic drainages or wadis occur in this region of KSA (Crosskey and Büttiker 1982, Alkahem and Behnke 1983, Whitton et al. 1986, Sorman and Abdulrazzak 1993, Al-Ghamdi and Abu-Zinadah 1998). These wadis are usually the only habitats for mayflies. Many of these drainages however, have been dammed to capture surface water. More than 230 dams now store annual runoff in reservoirs (Kingdom of Saudi Arabia Ministry of Foreign Affairs 2015). This water is used primarily for agriculture and is distributed through thousands of kilometers of irrigation canals, which can also support mayfly populations. Habitats such as oases (Edgell 2006) in KSA have not been adequately sampled for mayflies.

The first systematic studies of the mayflies of KSA were published more than 25 years ago. Six species belonging to three families (Baetidae, Caenidae and Leptophlebiidae) were mentioned from KSA (Sartori 1991; Sartori and Gillies 1990; Thomas and Sartori 1989). Within Baetidae, Baetis balcanicus Müller-Liebenau \& Soldán, 1981, Centroptilum dimorphicum Soldán \& Thomas, 1985 and Cloeon saharense Soldán \& Thomas, 1983 were reported (Sartori 1991; Sartori and Gillies 1990; Thomas and Sartori 1989). Baetis balcanicus is now considered as a Labiobaetis (Lugo-Ortiz and McCafferty 1997; McCafferty and Waltz 1995). The validity of this identification is debated below in the discussion section. Centroptilum dimorphicum is now assigned 
to the genus Cheleocloeon Wuillot \& Gillies, 1993 (Wuillot and Gillies 1993). The attribution of the specimens collected in the Arabian Peninsula to a species previously known only from North-West Africa appears now highly questionable, especially as a new species of Cheleocloeon, C. soldani Gattolliat \& Sartori, 2008, was described from United Arab Emirates (Gattolliat and Sartori 2008). We therefore consider the above specimens of Centroptilum dimorphicum reported from KSA as belonging to Cheleocloeon cf. soldani. Close morphological analysis is required before a definitive species assignment can be decided. At least two species of Cloeon Leach, 1815 occur in KSA and they appear both morphologically and genetically more related to Afrotropical species of Cloeon rather than to North African species such as Cloeon saharense (Salles et al. 2014).

\section{Material and methods}

The majority of the material was collected in November 2012 during a scientific expedition organised by King Saud University Museum of Arthropods, College of Food and Agriculture Sciences, Department of Plant Protection, King Saud University, KSA. Mayflies were collected from approximately 50 localities mainly along the southern coast of KSA. Other specimens were collected during different fieldtrips led by the same institution mainly in 2010 and 2012. Larvae were collected primarily by kick netting in stream vegetation along edges of wadis. Imagos were collected by light traps using black light with a white sheet. Mature larvae were reared in rearing chambers; larval and subimaginal exuviae were collected and stored with the corresponding imago. All material is stored in $80 \%$ ethanol. Holotypes and part of the paratypes are deposited in the King Saud University Museum of Arthropods, Riyadh, Saudi Arabia (KSU); other paratypes are housed in the Museum of Zoology, Lausanne, Switzerland (MZL). Each GBIFCH code refers to a tube with group of specimens in or a slide with a single specimen (sequenced or not).

The association of the ontogenetic stages was easily made for reared material. For specimens obtained by light trapping and kick sampling, we used sequence divergence of the fragment of mitochondrial cytochrome c oxidase subunit I (CO1) gene. Specimens belonging to the different "morphospecies" and collected in the same localities were selected for genetic analysis. The CO1 gene was sequenced using LCO1490 and HCO2198 primers (Folmer et al. 1994). We followed the laboratory procedures, edition and alignment of sequences as described in Vuataz et al. (2011). The final data matrix included $41 \mathrm{CO} 1$ sequences of $658 \mathrm{bp}$ representing all the Labiobaetis taxa from Palearctic and Afrotropical regions for which sequences are available (Table 1). Analyses were conducted in MEGA7 (Kumar et al. 2016).

Tree topology was reconstructed using the Maximum Likelihood method based on the Tamura-Nei model (Tamura and Nei 1993). The tree with the highest log likelihood is shown. The percentage of trees in which the associated taxa clustered together is shown next to the branches (Bootstrap with 1000 replicates). We used 3.5\% 
Table I. Specimens used for the phylogenetic analysis of the mitochondrial gene CO1.

\begin{tabular}{|c|c|c|c|c|}
\hline Species & Locality & Specimen catalog \# & $\begin{array}{c}\text { GenBank \# } \\
\text { (COI) }\end{array}$ & GenSeq Nomenclature \\
\hline \multirow{11}{*}{ Labiobaetis glaucus } & South Africa & GBIFCH00517537 & MH070310 & genseq-4 COI \\
\hline & South Africa & GBIFCH00517539 & MH070321 & genseq- 4 COI \\
\hline & South Africa & GBIFCH00517538 & MH070319 & genseq- 4 COI \\
\hline & Mayotte & GBIFCH00517531 & MH105069 & genseq-4 COI \\
\hline & Mayotte & GBIFCH00521580 & MH070315 & genseq- 4 COI \\
\hline & Mayotte & GBIFCH00517530 & MH070318 & genseq-4 COI \\
\hline & Saudi Arabia & GBIFCH00465151 & MH070288 & genseq- 4 COI \\
\hline & Saudi Arabia & GBIFCH00235741 & MH070311 & genseq-4 COI \\
\hline & Saudi Arabia & GBIFCH00235750 & MH105068 & genseq- 4 COI \\
\hline & Saudi Arabia & GBIFCH00235731 & MH070317 & genseq-4 COI \\
\hline & Saudi Arabia & GBIFCH00517523 & MH070320 & genseq-4 COI \\
\hline \multirow{11}{*}{$\begin{array}{l}\text { Labiobaetis potamoticus } \\
\text { sp. n. }\end{array}$} & Saudi Arabia & GBIFCH00517520 & MH070306 & genseq-2 COI \\
\hline & Saudi Arabia & GBIFCH00517521 & MH070308 & genseq-2 COI \\
\hline & Saudi Arabia & GBIFCH00235735 & MH070312 & genseq-2 COI \\
\hline & Saudi Arabia & GBIFCH00235732 & MH070316 & genseq-2 COI \\
\hline & Saudi Arabia & GBIFCH00465152 & MH070289 & genseq-2 COI \\
\hline & Saudi Arabia & GBIFCH00465154 & MH070290 & genseq-2 COI \\
\hline & Saudi Arabia & GBIFCH00517527 & MH070307 & genseq-2 COI \\
\hline & Saudi Arabia & GBIFCH00235747 & MH070313 & genseq-2 COI \\
\hline & Saudi Arabia & GBIFCH00235757 & MH070314 & genseq-2 COI \\
\hline & Saudi Arabia & GBIFCH00517526 & MH070322 & genseq-2 COI \\
\hline & Saudi Arabia & GBIFCH00465155 & MH070291 & genseq-2 COI \\
\hline Labiobaetis boussoulius & Ivory Coast & GBIFCH00517528 & MH070309 & genseq- 4 COI \\
\hline Labiobaetis sp. & Ivory Coast & GBIFCH00465136 & MH070294 & \\
\hline Labiobaetis sp. & Ivory Coast & GBIFCH00465137 & MH070295 & \\
\hline Labiobaetis sp. & Ivory Coast & GBIFCH00465138 & MH070296 & \\
\hline Labiobaetis sp. & South Africa & GBIFCH00465153 & MH070305 & \\
\hline Labiobaetis sp. & South Africa & GBIFCH00465135 & MH070303 & \\
\hline Labiobaetis piscis & South Africa & & $\begin{array}{c}\text { IBOL CED } \\
150 \mathrm{U}\end{array}$ & genseq-4 COI \\
\hline Labiobaetis latus & South Africa & GBIFCH00465142 & MH070297 & genseq- 4 COI \\
\hline Labiobaetis vinosus & South Africa & GBIFCH00465143 & MH070304 & genseq-4 COI \\
\hline Labiobaetis dambrensis & Madagascar & GBIFCH00465144 & MH070293 & genseq-2 COI \\
\hline Labiobaetis nigrocercus & Madagascar & GBIFCH00465145 & MH070300 & genseq- 4 COI \\
\hline \multirow{2}{*}{ Labiobaetis longicercus } & Madagascar & GBIFCH00465146 & MH070298 & genseq- 4 COI \\
\hline & Madagascar & GBIFCH00465147 & MH070299 & genseq- 4 COI \\
\hline \multirow{2}{*}{ Labiobaetis punctatus } & Madagascar & GBIFCH00465148 & MH070301 & genseq-4 COI \\
\hline & Madagascar & GBIFCH00465149 & MH070302 & genseq- 4 COI \\
\hline Labiobaetis atrebatinus & France & GBIFCH00465150 & MH070292 & genseq-4 COI \\
\hline L. atrebatinus orientalis & Japan & & KF563032 & genseq- 4 COI \\
\hline Labiobaetis tricolor & Hungary & & JN164313 & genseq-4 COI \\
\hline Baetis rhodani & Switzerland & & HG935037 & genseq-4 COI \\
\hline
\end{tabular}


sequence divergence (measured by Kimura 2-parameter (K2P)) as the maximal value for intraspecific divergence (Ball et al. 2005; Gattolliat et al. 2015; Rutschmann et al. 2014; Rutschmann et al. 2017; Webb et al. 2012). As part of the sequenced specimens came from the same population, the intraspecific divergence is expected to be even clearly lower than the limit.

\section{Taxonomy}

Labiobaetis potamoticus Gattolliat \& Al Dhafer, sp. n. http://zoobank.org/EAAD82B8-5680-49C0-879C-8B4F204FF351 Figs 1-19

Baetis balcanicus Müller-Liebenau \& Soldán, 1981 in Thomas and Sartori 1989: 87.

Type material. Holotype: Male larva (GBIFCH00521578): Saudi Arabia (AR44); Wadi Shahadan; 17²8'36"/ 4242'50"; Alt. 190m; 13.XI.2012; Coll. J-L Gattolliat.

Paratypes: 4 larvae (GBIFCH00235716 + GBIFCH00235735 (Genetics)), 1 male imago (GBIFCH00235732 (Genetics)) + 3 larvae (KSU: GBIFCH00526192); same data as holotype.

42 larvae (GBIFCH00235729 + 3 slides GBIFCH00235758, GBIFCH00235733, GBIFCH00235760): Saudi Arabia (AR01); Al Jiwah, Thee Aine; 1955'32"/ 4126'17"; Alt. 752m; 13.X.2010; Coll. B. Kondratieff.

14 larvae (GBIFCH00235721): Saudi Arabia (AR20); Wadi Baqrah; 1847'29"/ 4156'19"; Alt. 490m; 13.III.2012; Coll. Al Dhafer, H. \& Kondratieff, B.

5 larvae (GBIFCH00235722): Saudi Arabia (AR28); Thee Ain, Al-Baha; 1955'46"/ 4126'34"; Alt. 760m; 3.VI.2012; Coll. Al Dhafer, H. \& Kondratieff, B.

2 larvae (GBIFCH00235714): Saudi Arabia (AR31); Thee Ain, Al-Baha; 1955'46"/ 41²6'34"; Alt. 760m; 8.XI.2012; Coll. J-L Gattolliat.

58 larvae (GBIFCH00235706, GBIFCH00235728, GBIFCH00235717 + 2 slides GBIFCH00235749, GBIFCH00235759 + GBIFCH00517520 (Genetics), GBIFCH00517521 (Genetics)) + 11 larvae (KSU: GBIFCH00526173): Saudi Arabia (AR32); Wadi Elarj, near Adam; 20²7'11"/ 4048'56"; Alt. 440m; 9.XI.2012; Coll. J-L Gattolliat.

23 larvae (GBIFCH00235726, GBIFCH00235719 + 3 slides GBIFCH00235753, GBIFCH00465152 (Genetics), GBIFCH00465155 (Genetics)) + 4 larvae (KSU: GBIFCH00526224): Saudi Arabia (AR43a); Wadi Shahadan; 17²8'36"/ 4251'25"; Alt. 460m; 12.XI.2012; Coll. J-L Gattolliat.

3 larvae (KSU: GBIFCH00526237): Saudi Arabia (AR43b); Wadi Shahadan; 17²8'17"/ 4251'14"; Alt. 455m; 12.XI.2012; Coll. J-L Gattolliat.

Additional material. 1 larva (on slide), Saudi Arabia, Wadi Buwah, $1340 \mathrm{~m}$, $20^{\circ} 47^{\prime} \mathrm{N} / 41^{\circ} 12^{\prime} \mathrm{E}$, 20.IX.1980, Leg. W. Büttiker. Coll. Naturhistorisches Museum Basel, Switzerland. (Previously identified as Baetis balcanicus, det. A. Thomas). 
Differential diagnosis. Larva: Tergites I-X medium brown with peculiar pattern formed of six ecru dots (Fig. 19). Scape of antenna without distolateral process (Fig. 13). Segment II of the maxillary palp without a distomedial concavity (Fig. 7). Segment II of labial palp with a slender triangular distomedial projection (Fig. 8). Dorsal margin of femur with a few medium setae, not abundant proximally; ventral margin with abundant medium setae (Fig. 10). Dorsal margin of tibia almost bare (Fig. 9). Paraproct with numerous distal spines (Fig. 15). Male imago: Genitalia with inner margin of segments I and II without expansion; segment III almost globular (Fig. 18).

Description. Larva. Length: fully grown female: Body $5.1-7.7 \mathrm{~mm}$, cerci $3.6-4.0$ $\mathrm{mm}$, terminal filament $2.5-2.6 \mathrm{~mm}$. Fully grown male: Body $5.0-5.3 \mathrm{~mm}$, cerci $3.4-$ $3.6 \mathrm{~mm}$, terminal filament $2.5 \mathrm{~mm}$.

Colouration (Fig. 19): Head almost uniformly brown with ecru vermiform marking on vertex and frons, border of sclerites yellow. Prothorax medium brown, lighter laterally; mesothorax medium brown with a central yellow dot and a transversal yellow stripe; metathorax medium brown. Legs: ecru except a brown central dot on femora; dorsal and ventral margin of femora brown, dorsal margin of tibiae and tarsi brown. Tergites I-X medium brown with peculiar pattern formed of six ecru dots sometimes fused. Abdominal sternites brown sometimes with four ecru dots. Cerci ecru brown getting progressively ecru towards apex.

Head: scape of antenna without distolateral process (Fig. 13).

Labrum (Fig. 1) rounded with a small anteromedial emargination, dorsally with a distolateral row of approx. eight feathered setae, without a submedian seta; short, thin, simple setae scattered on dorsal surface of labrum; distal margin bordered with feathered setae.

Right mandible (Fig. 2): canine with two almost fused incisivi each with four denticles, outer denticle much shorter than others, inner margin of inner incisive with a row of very thin setae; stout prostheca apically with small pointed denticles (Fig. 3); margin between prostheca and mola slightly convex, smooth, without setae; tuft of setae at apex of mola absent.

Left mandible (Fig. 4): canine with two almost fused incisivi each with four denticles, outer denticle much shorter than others; stout prostheca apically with small denticles and a comb-shaped structure (Fig. 5); margin between prostheca and mola slightly concave, without crenulations; tuft of setae at apex of mola absent.

Hypopharynx as illustrated in Fig. 6.

Maxilla (Fig. 7) with a medioapical row of medium setae, basal end of row with a few long setae; posterior side of lacinia mediobasally with a row of four medium-sized setae, a single small seta close to the medial margin of lacinia; palp 2-segmented, longer than galea-lacinia, segment II without distomedial concavity.

Labium (Fig. 8) with glossae shorter than paraglossae; glossae inner margin with two rows of approx. six long setae, apically with a few simple setae; paraglossae stout, apically flattened, with three rows of long simple setae; labial palp with segment I slender, quadrangular, shorter than segments II and III combined; segment II with a 

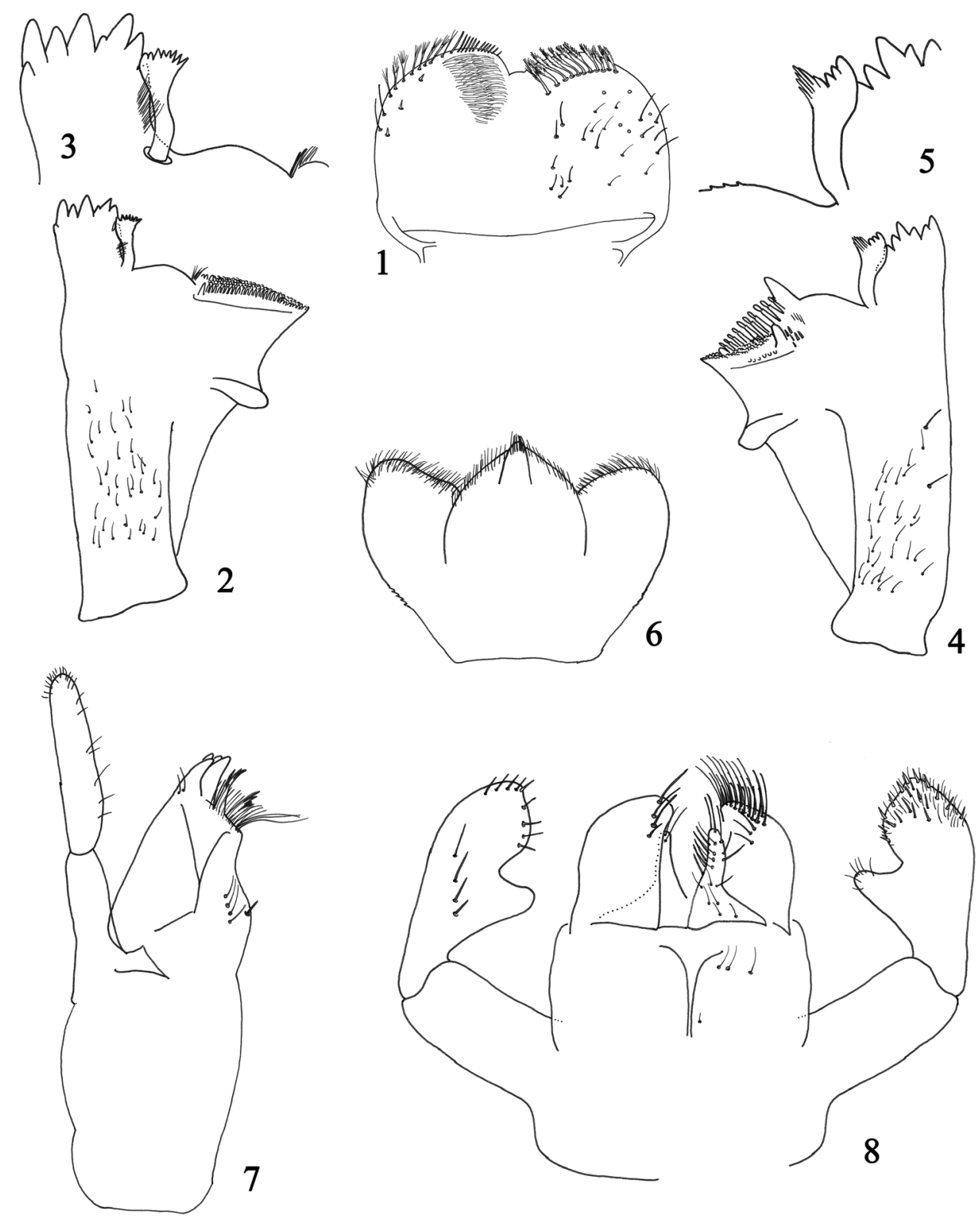

Figures I-8. Larval structures of Labiobaetis potamoticus sp. n.: I labrum (left : ventral; right : dorsal) $\mathbf{2}$ right mandible $\mathbf{3}$ detail of right mandible: canines and prostheca $\mathbf{4}$ left mandible $\mathbf{5}$ detail of left mandible: prostheca $\mathbf{6}$ hypopharynx $\mathbf{7}$ right maxilla $\mathbf{8}$ labium.

slender, elongated distomedial projection with few thin setae apically, on posterior side with a row of four long setae increasing in length; segment III subconical, inner margin apically slightly concave, with abundant scattered short thin setae and stouter setae. 

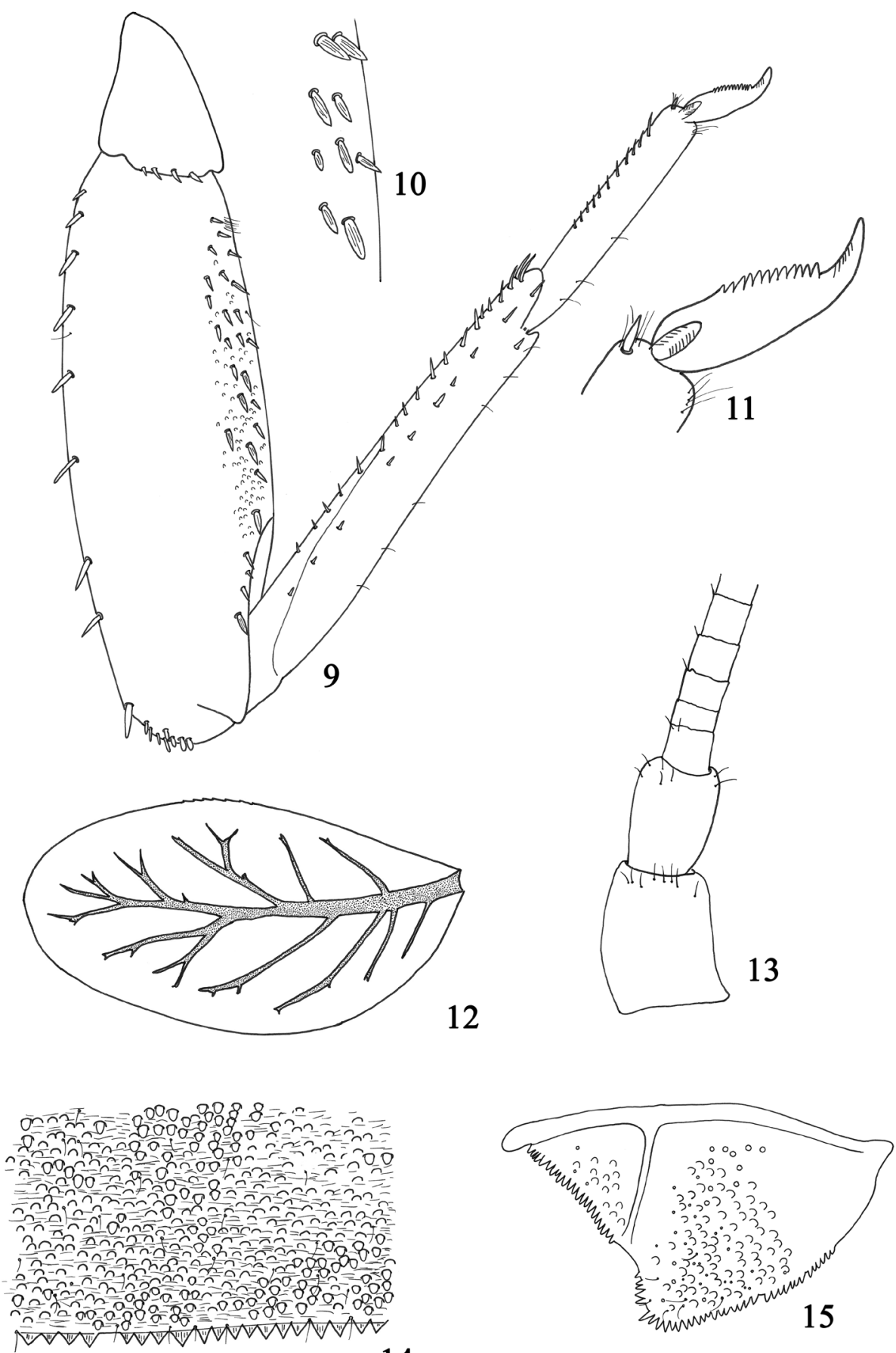

Figures 9-I5. Larval structures of Labiobaetis potamoticus sp. $\mathrm{n} .: \mathbf{9}$ foreleg $\mathbf{I 0}$ detail of ventral margin of forefemora I I tarsal claw $\mathbf{I} \mathbf{2}$ fourth gill $\mathbf{I} \mathbf{3}$ base of antenna $\mathbf{4}$ posterior margin of fourth abdominal tergite $\mathbf{I} \mathbf{5}$ paraproct. 

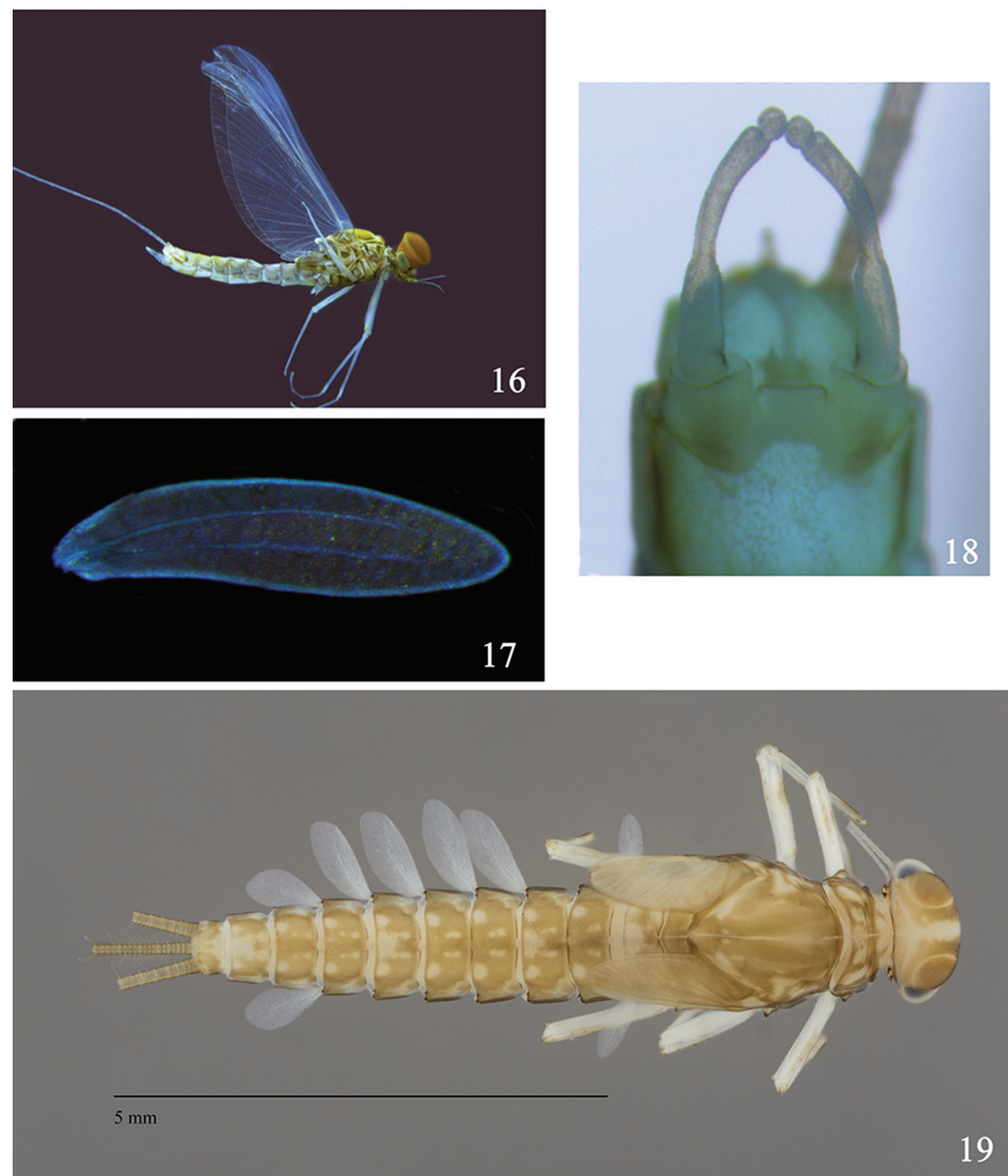

Figures 16-19. Larval and imaginal structures of Labiobaetis potamoticus sp. n.: 16 male imago (lateral view) $\mathbf{1}$ hindwing $\mathbf{8}$ male genitalia $\mathbf{9}$ male larva (dorsal view).

Thorax: hind wing pads present.

Legs (Fig. 9): forefemur dorsally with a row of approx. eight medium-sized, apically rounded setae; apex with one medium stout seta and several short flattened setae; ventral margin with a poorly developed villopore and abundant, medium, stout setae (Fig. 10). Foretibia dorsally almost bare; ventrally with short setae, only slightly longer apically. Foretarsus almost bare dorsally; ventral margin with a row of pointed setae slightly increasing in length toward apex; tarsal claw (Fig. 11) with a single row of ap- 
prox. twelve pointed teeth; without subapical setae. Middle and hind legs similar to foreleg but with reduced setation.

Abdomen: tergites (Fig. 14) shagreened with numerous scales and scale bases, with a few setae; posterior margin with triangular spination as long as broad. Sternites with scales and scale bases; posterior margin smooth, without spines.

Gills present on abdominal segments I-VII (Fig. 12), poorly serrated, tracheation brown, with abundant ramifications.

Paraproct (Fig. 15) with scale bases and a few setae, margin with numerous small triangular spines regular in size; posterolateral extension with a few scale bases, spines along the margin of the same size as those of the paraproct.

Male imago

Length. Body $4.8 \mathrm{~mm}$; forewing $4.4 \mathrm{~mm}$; hindwing $0.8 \mathrm{~mm}$.

Colouration: head brown; antenna ecru except base of scape and pedicel brown. Facetted surface of turbinate eyes dark orange brown, shaft orange brown, lighter apically (Fig. 16). Thorax yellowish brown with margin of sclerites generally dark brown. Legs: yellowish without marks or pattern. Wings hyaline, hyaline venation. Abdomen: tergites I to X ecru without pattern. Sternites I to IX ecru. Cerci ecru. Genitalia (Fig. 18) ecru except inner margin of segment I medium brown.

Forewing: pterostigma with approx. two cross-veins not reaching subcostal vein; double intercalary veins shorter than distance between corresponding main veins.

Hindwing (Fig. 17) without costal spur; two longitudinal veins almost reaching margin, none of them bifurcated, without incomplete veinlets between main longitudinal veins.

Genitalia (Fig. 18): basal segment with inner margin not expanded apically; segments I and II almost completely fused; constriction at basis of segment II; segment III globular.

Distribution and habitat. Labiobaetis potamoticus appears to be the most widespread species of Labiobaetis in KSA. It colonizes aquatic vegetation in still reaches of small to medium-sized streams with a sandy substrate (Figs 48, 52). This species was also collected in a secondary channel with Typha sp. (Fig. 49). This species seems less rheophilic than the two following taxa.

Etymology. The specific name potamic- was given in reference to the ecological preferences of the species for still and standing water.

\section{Labiobaetis alahmadii Gattolliat \& Al Dhafer, sp. n.}

http://zoobank.org/605BFF9A-A54E-419F-912B-462B1E9FAAF9

Type material. Holotype: Female larva (GBIFCH00521579): Saudi Arabia (AR43a); Wadi Shahadan; 17²8'36"/ 4251'25"; Alt. 460m; 12.XI.2012; Coll. J-L Gattolliat.

Paratypes: 151 larvae (GBIFCH00235715, GBIFCH00235720 + GBIFCH00517525 + 3 slides GBIFCH00235737, GBIFCH00235755, GBIFCH00235757 (Genetics), GBIFCH00235737) + 8 larvae (KSU: GBIFCH00526208): same data as holotype. 
47 larvae (GBIFCH00235710) + 10 larvae (KSU: GBIFCH00526227): Saudi Arabia (AR43b); Wadi Shahadan; 17²8'17"/ 4251'14"; Alt. 455m; 12.XI.2012; Coll. J-L Gattolliat.

29 larvae (GBIFCH00235727) + 6 larvae (KSU: GBIFCH00526223): Saudi Arabia (AR39); Wadi Damad; 17²1'21"/ 4301'35"; Alt. 260m; 11.XI.2012; Coll. J-L Gattolliat.

15 larvae (GBIFCH00235709 + 3 slides GBIFCH00235744, GBIFCH00235747 (Genetics), GBIFCH00465155 (Genetics) + GBIFCH00517526 (Genetics), GBIFCH00517527 (Genetics)) + 4 larvae (KSU: GBIFCH00526179): Saudi Arabia (AR44); Wadi Shahadan; 17²8'36"/ 4242'50"; Alt. 190m; 13.XI.2012; Coll. J-L Gattolliat.

Differential diagnosis. Larva: Colouration: mesothorax medium brown with a W-shaped yellow pattern; tergites I-VIII medium brown with two broad ecru spots, tergites IX and X yellow (Figs 32, 33). Scape of antenna without distolateral process (Fig. 28). Segment II of the maxillary palp with a small distomedial concavity (Fig. 23). Segment II of labial palp with a broad apically rounded triangular distomedial projection (Fig. 24). Dorsal margin of femur with regularly spaced setae; ventral margin almost bare (Fig. 25). Dorsal margin of tibia with a row of small spatulate setae (Fig. 26). Paraproct with approximately eight stout, pointed spines increasing in length toward the apex (Fig. 31).

Description. Larva. Length: fully grown female: Body $9.5-10.6 \mathrm{~mm}$, cerci 4.0 $4.2 \mathrm{~mm}$, terminal filament 2.9-3.1 mm. Fully grown male: Body 8.8-9.9 mm, cerci $3.8-4.1 \mathrm{~mm}$, terminal filament $2.8-2.9 \mathrm{~mm}$.

Colouration (Figs 32, 33): head almost uniformly medium brown, with darker, vermiform marking on vertex and frons, border of sclerites yellow. Prothorax ecru with proximal margin medium brown and a brown dot medio-apically; mesothorax medium brown with a double $\mathrm{V}$-shaped yellow pattern; metathorax medium brown. Legs: ecru except femora with a central brown spot and apex of femora, tibiae and tarsi brown. Tergites I-VIII medium brown with two broad ecru spots sometimes fused medially, tergites IX and X yellow. Abdominal sternites ecru getting darker and brownish after sternite VI. Cerci ecru without dark stripe.

Head: scape of antenna without distolateral process (Fig. 28).

Labrum (Fig. 20) rounded, with a small anteromedial emargination, dorsally with a relatively short submedian seta and a distolateral row of approx. ten feathered setae; short, thin, simple setae scattered on dorsal surface of labrum; distal margin bordered with feathered setae.

Right mandible (Fig. 21): canine with two almost fused incisivi each with four denticles, outer denticle much shorter than others, inner margin of inner incisive with a row of very thin setae; stout prostheca apically with numerous small rounded denticles, also covering distoapical corner; margin between prostheca and mola slightly convex, smooth, without setae; tuft of setae at apex of mola present.

Left mandible (Fig. 22): canine with two almost fused incisivi each with four denticles, outer denticle much shorter than others; stout prostheca apically with small den- 

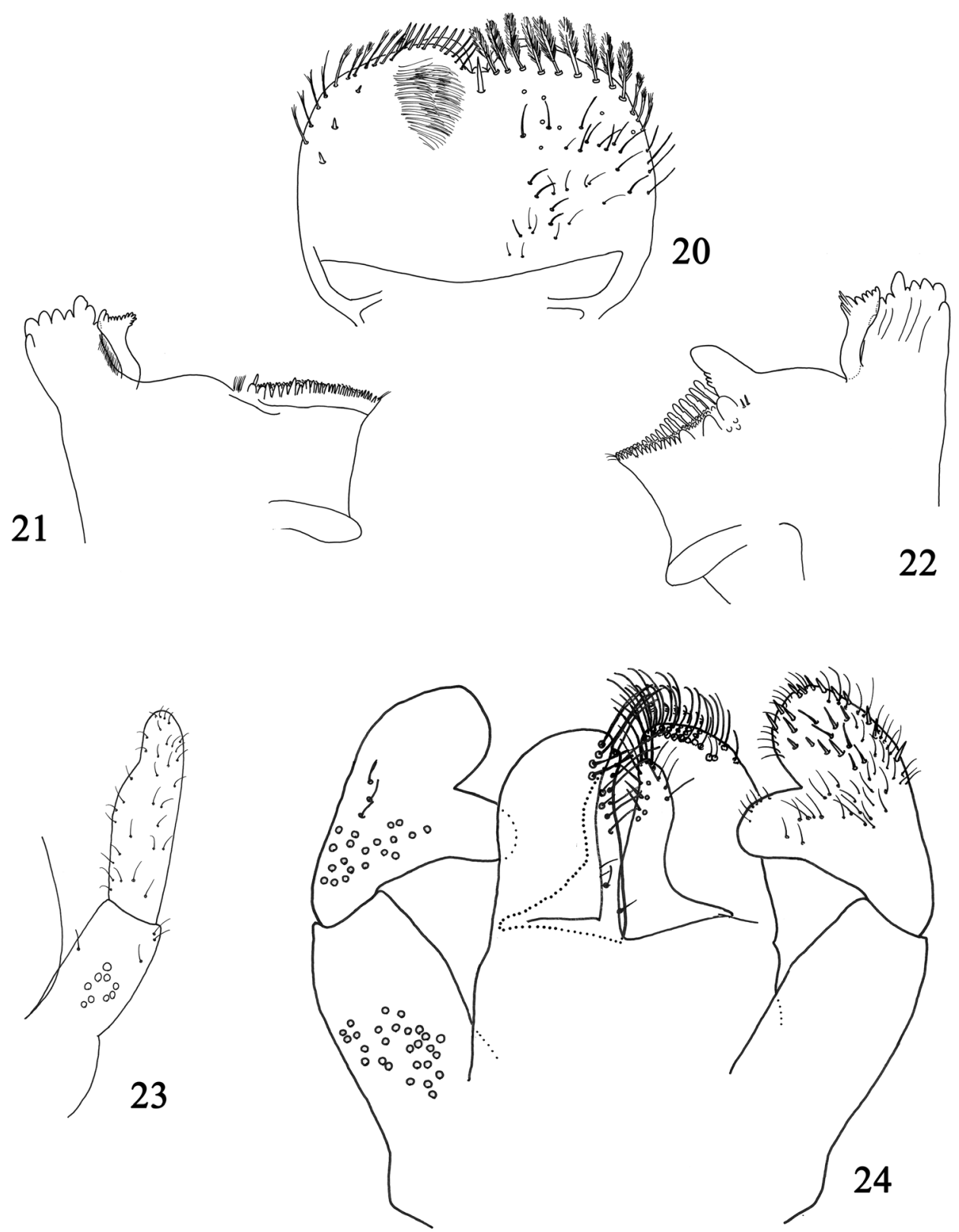

Figures 20-24. Larval structures of Labiobaetis alahmadii sp. n.: 20 labrum (left : ventral; right: dorsal) $\mathbf{2 1}$ right mandible $\mathbf{2 2}$ left mandible $\mathbf{2 3}$ detail of maxilla $\mathbf{2 4}$ labium.

ticles and a comb-shaped structure; margin between prostheca and mandible slightly concave, without crenulations; tuft of setae at apex of mola present.

Hypopharynx similar to Fig. 6. 

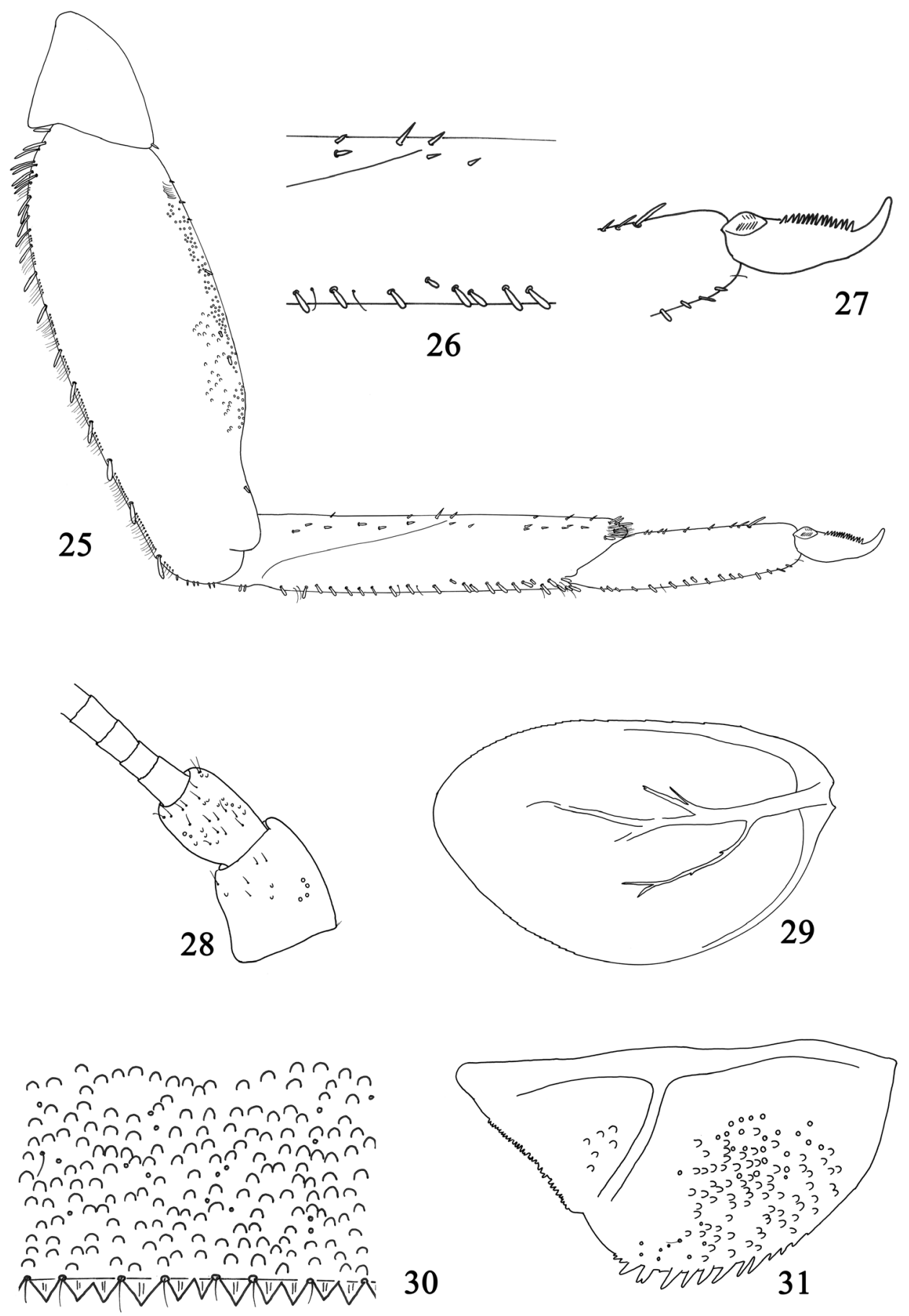

Figures 25-3 I. Larval structures of Labiobaetis alahmadii sp. n.: $\mathbf{2 5}$ foreleg $\mathbf{2 6}$ detail of ventral margin of foretibia $\mathbf{2 7}$ tarsal claw $\mathbf{2 8}$ base of antenna $\mathbf{2 9}$ fourth gill $\mathbf{3 0}$ posterior margin of fourth abdominal tergite $\mathbf{3} \mathbf{I}$ paraproct. 

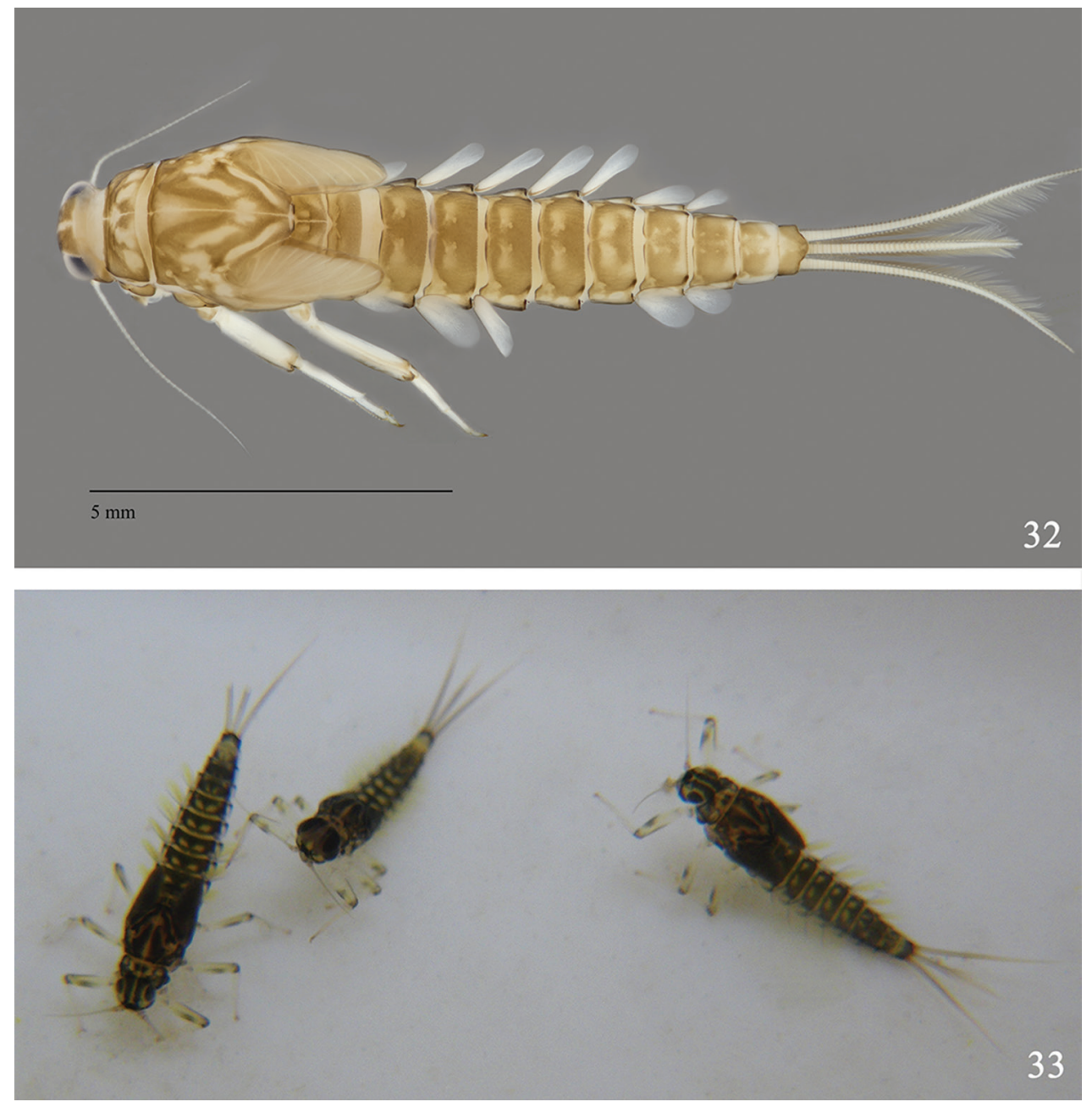

Figures 32-33. Larvae of Labiobaetis alahmadii sp. n.: 32 female larva (dorsal view) 33 larvae in vivo.

Maxilla with a medioapical row of medium setae, basal end of row with a few long setae; posterior side of lacinia mediobasally with a row of four medium-sized setae, a single small seta close to the medial margin of lacinia; palp 2-segmented, as long as galea-lacinia, segment II with distomedial concavity (Fig. 23).

Labium (Fig. 24) with glossae shorter than paraglossae; glossae with medium to long simple setae in apical half; paraglossae stout, apically rounded, with three rows of setae: two rows with simple setae and one row with setae feathered on one side; labial palp with segment I slender, quadrangular, as long as segments II and III combined; segment II with an elongated triangular distomedial projection with few scattered thin setae, on posterior side with a row of three medium setae; segment III subconical, with abundant scattered short thin setae and stouter setae. 
Thorax: hind wing pads present.

Legs (Fig. 25): forefemur dorsally with a row of medium-sized, apically rounded setae, abundant proximally; apex with one short stout seta and minute setae; ventral margin with a poorly developed villopore and scarce minute setae. Foretibia dorsally with a row of short spatulate setae (Fig. 26); ventrally with short setae, not longer apically, apex with a patch of numerous flattened short setae. Foretarsus dorsally with a row of short spatulate setae; ventral margin with a row of minute pointed setae slightly increasing in length toward apex; tarsal claw (Fig. 27) with a single row of approx. 14 pointed teeth; without subapical setae. Middle and hind legs similar to foreleg but with reduced setation.

Abdomen: tergites (Fig. 30) with numerous scale bases with a few setae; posterior margin with triangular spination as long as broad. Sternites with numerous scales and scale bases; sternites I-VII with posterior margin smooth without spines, sternites VIII and IX with small triangular spines.

Gills present on abdominal segments I-VII, margins serrated, tracheation poorly marked and poorly divided (Fig. 29).

Paraproct (Fig. 31) with scale bases, almost bare, margin with approx. eight stout, pointed spines and bordered by few small spines; posterolateral extension with a few scale bases, minute spines along the margin.

Imagos: Unknown

Distribution and habitat. We collected this species in only three different localities in close proximity, in extreme southwestern KSA close to the Yemen border. The larvae occur in medium-size streams with stony substrates (Figs 50, 51, 52). Larvae apparently prefer relatively fast flowing reaches. Larvae were even collected at the base of small waterfalls. Labiobaetis alahmadii appeared to be an abundant species when the above ecological conditions are optimal. The new species was always sympatric with L. glaucus.

Etymology. This species is dedicated to the memory of Professor Ahmed Ziad Al Ahmadi, the well-known Syrian entomologist who passed away few months ago.

\section{Labiobaetis glaucus (Agnew, 1961)}

Figs 34-47

Baetis glaucus Agnew, 1961: 14.

Pseudocloeon glaucum, Lugo-Ortiz et al. 2000: 281.

Labiobaetis glaucus, Kluge and Novikova 2016: 32-33.

Specimens examined. 18 larvae (GBIFCH00235711 + 4 slides GBIFCH00235741 (Genetics), GBIFCH00235746, GBIFCH00235750 (Genetics), GBIFCH00235756: Saudi Arabia (AR01); Al Jiwah, Thee Aine; 1955'32"/ 4126'17"; Alt. 752m; 13.X.2010; Coll. B. Kondratieff. 
3 larvae (GBIFCH00235708): Saudi Arabia (AR19); Wadi Khat; 1905'22"/ 4158'16"; Alt. 490m; 13.III.2012; Coll. Al Dhafer, H.

1 larva (GBIFCH00235712): Saudi Arabia (AR28); Thee Ain, Al-Baha; 1955'43"/ 4126'34"; Alt. 760m; 3.VI.2012; Coll. Al Dhafer, H. \& Kondratieff, B.

3 larvae (GBIFCH00235713): Saudi Arabia (AR31); Thee Ain, Al-Baha; 1955'43"/ 41²6'34"; Alt. 760m; 8.XI.2012; Coll. J-L Gattolliat.

1 larva (GBIFCH00235707): Saudi Arabia (AR43a); Wadi Shahadan; 17²8'36"/ 4251'25"; Alt. 460m; 12.XI.2012; Coll. J-L Gattolliat.

2 larvae GBIFCH00465151 (Genetics): Saudi Arabia (AR43b); Wadi Shahadan; $17^{\circ} 28^{\prime} 17^{\prime \prime} / 42^{\circ} 51^{\prime} 14 "$ "; Alt. 440m; 12.XI.2012; Coll. J-L Gattolliat.

7 larvae (GBIFCH00235723 + 1 slide GBIFCH00235738), 3 male imagos (GBIFCH00235724 + 1 slide GBIFCH00235731 (Genetics)): Saudi Arabia (AR44); Wadi Shahadan; 17²8'36"/ 4242'50"; Alt. 190m; 13.XI.2012; Coll. J-L Gattolliat.

Differential diagnosis. Larva: abdominal pattern (Fig. 47) with tergites I, VI and $\mathrm{X}$ lighter (in some specimens tergites $\mathrm{V}$ and IX also lighter). Scape of antenna without distolateral process (Fig. 41). Segment II of the maxillary palp without a distomedial concavity (Fig. 38). Segment II of labial palp with a broad apically rounded triangular distomedial projection (Fig. 39). Dorsal margin of femur (Fig. 40) with numerous setae proximally and rarely any distally; ventral margin with a few scattered setae. Dorsal margin of tibia with a few minute setae. Paraproct with approx. ten stout, pointed spines increasing in length towards apex (Fig. 44). Male imago: Genitalia with inner margin at the apex of segment I and base of segment II with a triangular well-marked expansion; segment III almost globular (Fig. 46).

Description. Larva. Length: fully grown female: Body $6.2-8.0 \mathrm{~mm}$, cerci 3.6$4.0 \mathrm{~mm}$, terminal filament $2.4-2.8 \mathrm{~mm}$. Fully grown male: Body $4.8-7.3 \mathrm{~mm}$, cerci 3.3-3.6 mm, terminal filament $1.7-1.9 \mathrm{~mm}$.

Colouration (Fig. 47): head almost uniformly medium brown, with darker, faint vermiform marking on vertex and frons, border of sclerites yellow. Prothorax medium brown with poorly marked yellowish pattern; mesothorax medium brown with a Vshaped yellow pattern; metathorax medium brown. Legs ecru except femora with a central brown spot and apex of femora, tibiae and tarsi brown. Tergites medium brown with small ecru spot except tergites I, V, VI, IX and X yellow, tergites V and VI generally with a dark M-shaped mark. Abdominal sternites ecru. Cerci ecru without dark stripe.

Head: scape of antenna without distolateral process (Fig. 41).

Labrum (Fig. 34) rounded, with a small anteromedial emargination, dorsally with one feathered submedian seta, and a distolateral row of approx. eight feathered setae; short, thin, simple setae scattered on dorsal surface of labrum; distal margin bordered with feathered setae.

Right mandible (Fig. 35): canine with two almost fused incisivi each with four denticles, outer denticle much shorter than others, inner margin of inner incisive with a row of very thin setae; stout prostheca apically with small rounded denticles; margin between prostheca and mola straight, smooth, without setae; tuft of setae at apex of mola reduced to two small setae. 

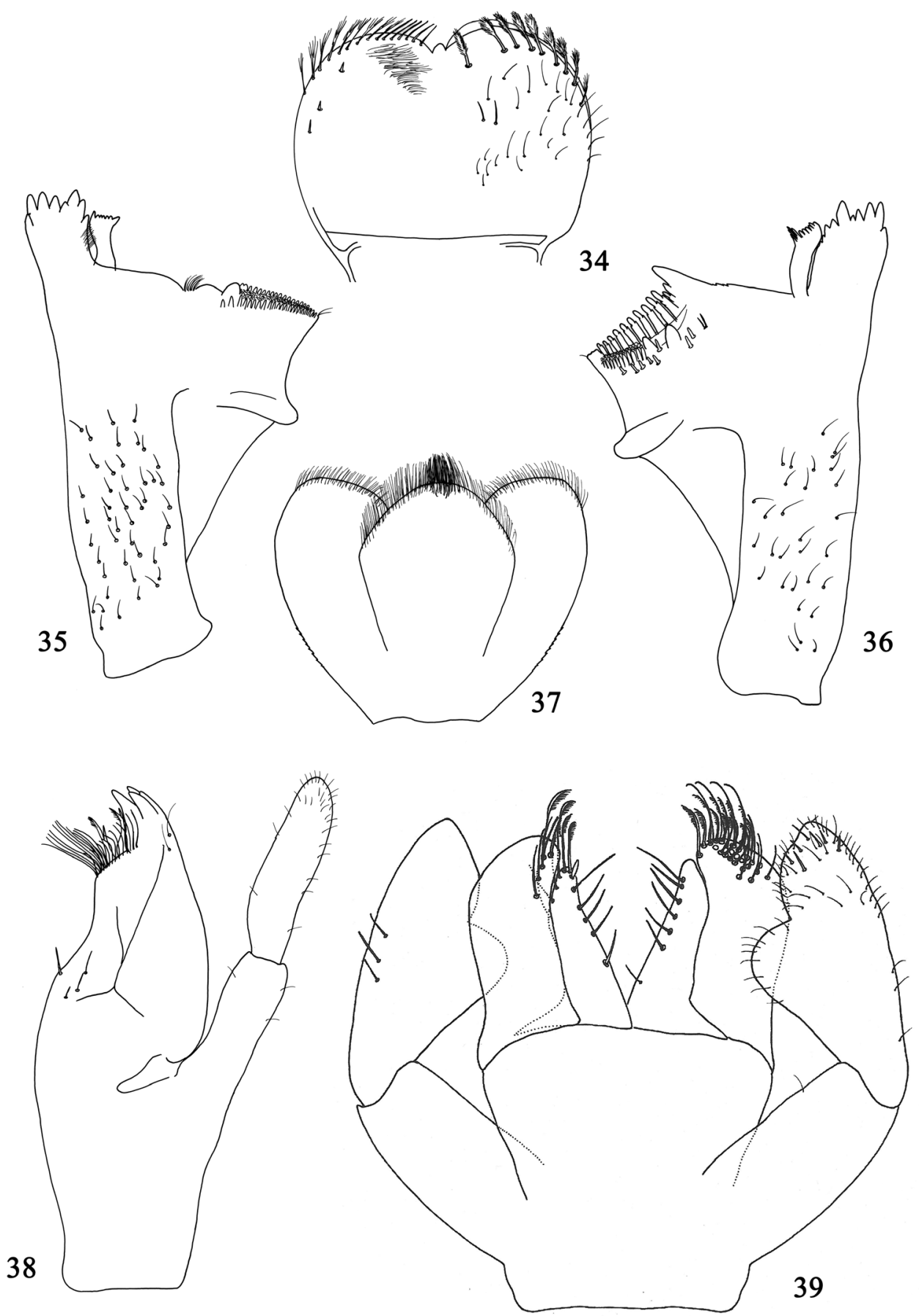

Figures 34-39. Larval structures of Labiobaetis glaucus: 34 labrum (left: ventral; right: dorsal) 35 right mandible $\mathbf{3 6}$ left mandible $\mathbf{3 7}$ hypopharynx 38 left maxilla $\mathbf{3 9}$ labium. 

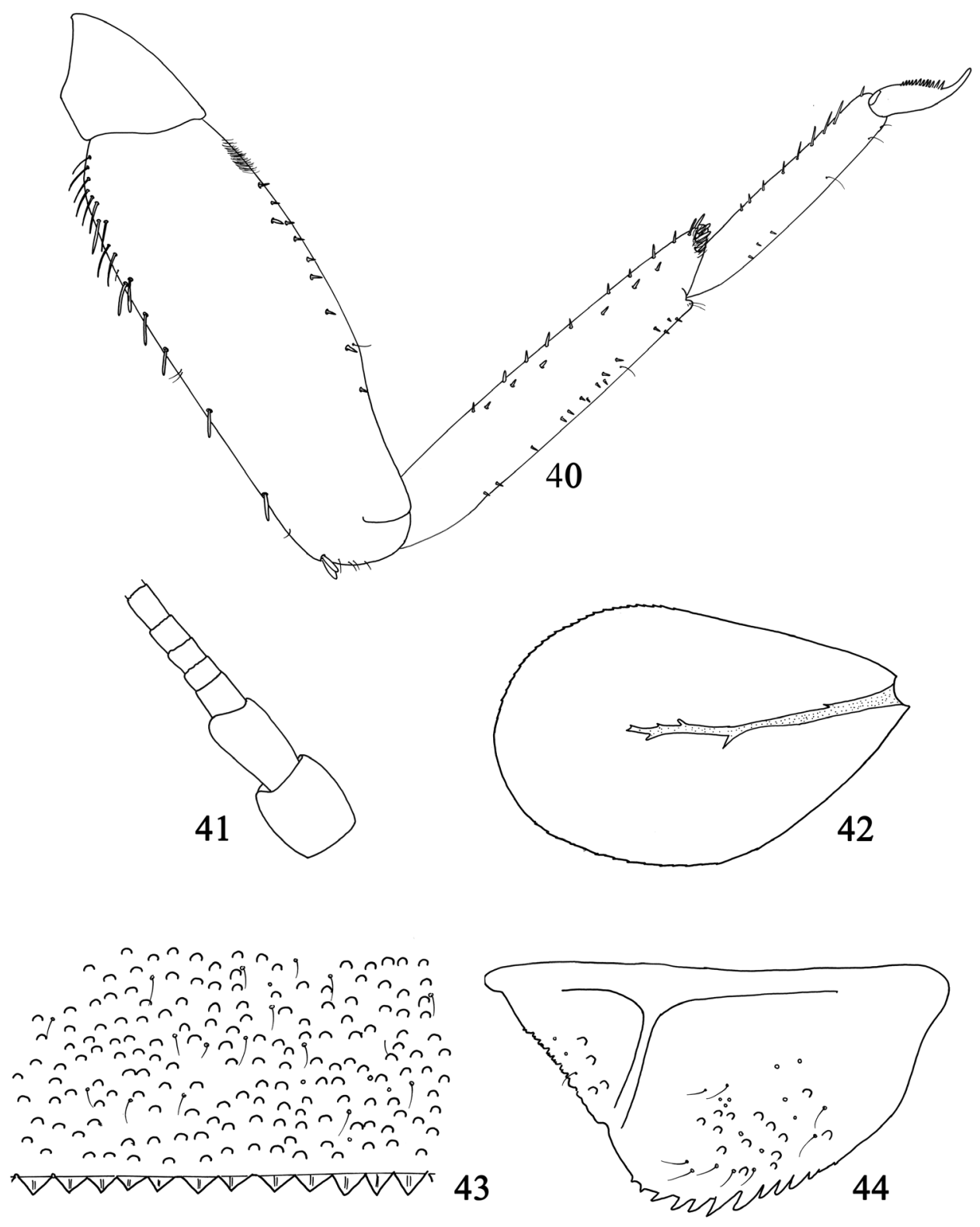

Figures 40-44. Larval structures of Labiobaetis glaucus: $\mathbf{4 0}$ foreleg $\mathbf{4 I}$ base of antenna $\mathbf{4 2}$ fourth gill $\mathbf{4 3}$ posterior margin of fourth abdominal tergite $\mathbf{4 4}$ paraproct.

Left mandible (Fig. 36): canine with two almost fused incisivi each with four denticles, outer denticle much shorter than others; stout prostheca apically with small denticles and a comb-shaped structure; margin between prostheca and mola straight, distally with crenulations; tuft of setae at apex of mola reduced to a single seta. 

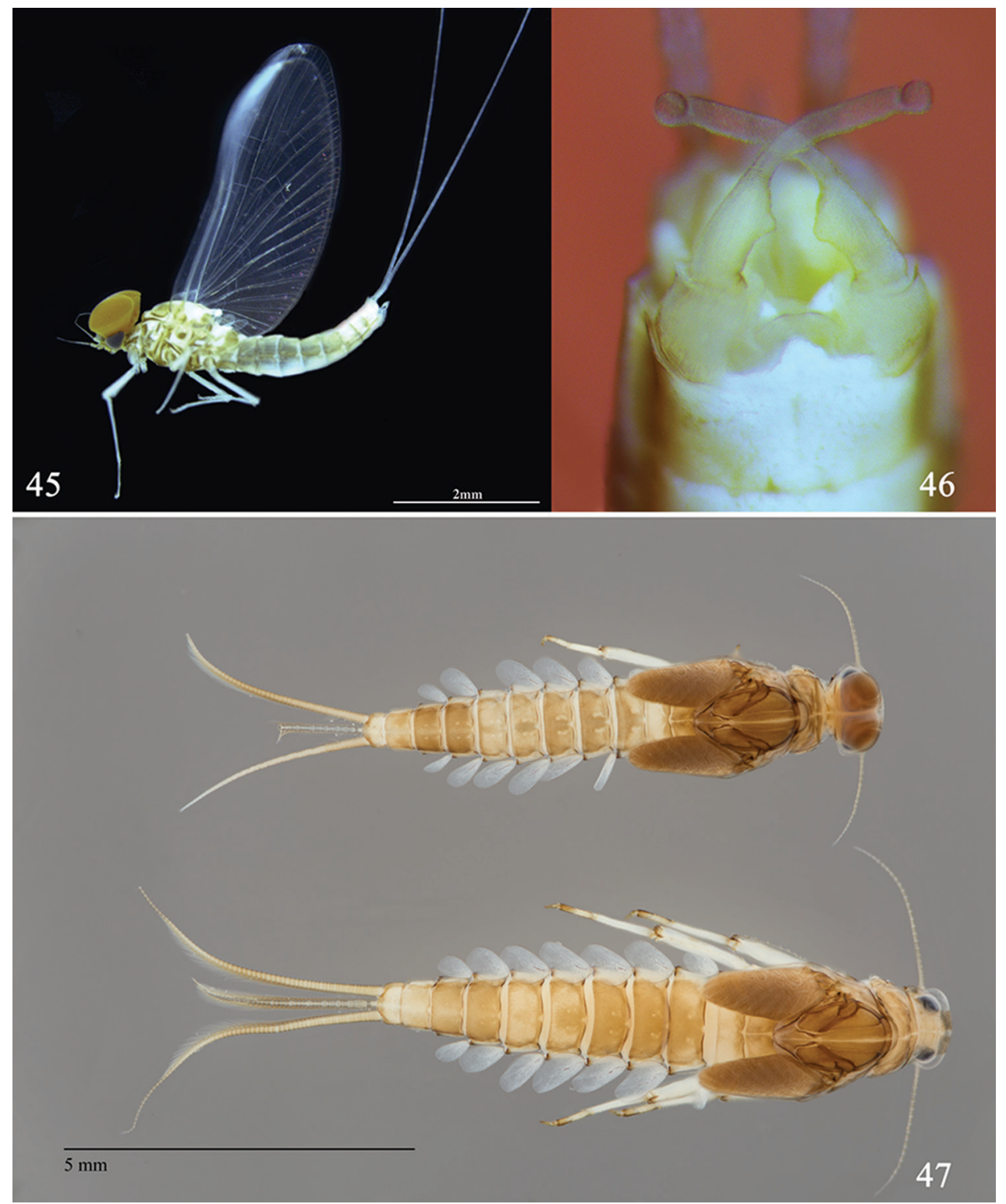

Figures 45-47. Larval and imaginal structures of Labiobaetis glaucus: 45 male imago (lateral view) 46 male genitalia 47 male and female larvae (dorsal view).

Hypopharynx as in Fig. 37.

Maxilla (Fig. 38) with a medioapical row of relatively short setae, basal end of row with approx. seven long setae; posterior side of lacinia mediobasally with a row of three medium-sized setae, a single small seta close to the medial margin of lacinia; palp 2-segmented, segment II without distomedial concavity. 

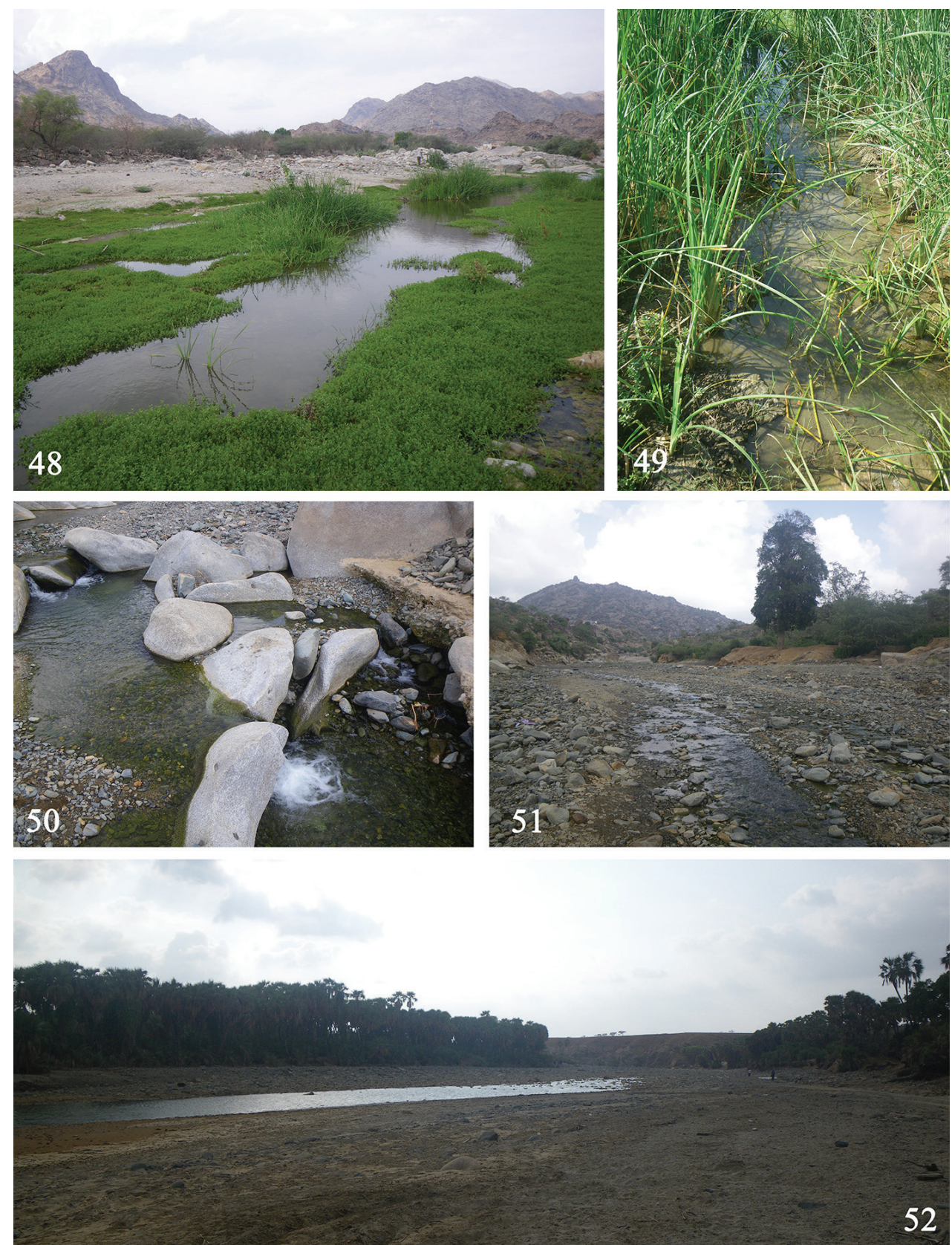

Figures 48-52. General aspects of Labiobaetis larval habitats: 48 AR32; Wadi Elarj, near Adam (Type locality of Labiobaetis potamoticus) 49 AR32; Wadi Elarj, near Adam: lateral channel with Typha sp. 50 AR43b; Wadi Shahadan: small waterfall 5 I AR43a; Wadi Shahadan (type locality of Labiobaetis alahmadii) 52 AR44; Wadi Shahadan. 
Labium (Fig. 39) with glossae slightly shorter than paraglossae; glossae inner margin with two rows of approx. six long setae, apically with a few setae feathered on one side; paraglossae stout, apically rounded, with three rows of long setae, part of them feathered on one side; labial palp with segment I slender, shorter than segments II and III combined; segment II with a broad apically rounded triangular distomedial projection covered with thin setae, on posterior side with a row of three long setae; segment III subconical, inner margin apically slightly concave, with scattered short thin setae and a few stouter setae.

Thorax: hind wing pads present.

Legs (Fig. 40): Forefemur dorsally with a row of medium-sized, apically rounded setae, numerous proximally and rare distally; apex with two short flattened setae; ventral margin with a well-developed villopore and scarce, short, stout setae. Foretibia dorsally with a row of scarce tiny, stout setae; ventrally with a few short setae, not longer apically, apex with a patch of numerous flattened short setae. Foretarsus almost bare dorsally; ventral margin with a row of pointed setae slightly increasing in length toward apex; tarsal claw with a single row of approx. twelve pointed teeth; subapical setae absent. Middle and hind legs similar to foreleg but with reduced setation.

Abdomen: tergites (Fig. 43) with numerous scale bases, with a few setae; posterior margin with short and broad triangular spination. Sternites with a few setae, without scales and scale bases; sternites I-VII with posterior margin smooth without spines, sternites VIII and IX with small triangular spines.

Gills present on abdominal segments I-VII, distally serrated, tracheation brown, poorly developed (Fig. 42).

Paraproct (Fig. 44) with scale bases and a few setae, margin with approx. ten stout, pointed spines increasing in length; posterolateral extension with a few scale bases, minute spines along the margin.

Male imago. Length. Body 4.4-4.5 mm; forewing 4.2-4.3 mm; hindwing $0.8 \mathrm{~mm}$.

Colouration: head dark brown; antenna ecru. Facetted surface of turbinate eyes orange brown, shaft orange brown (Fig. 45). Thorax yellowish brown with margin of sclerites generally dark brown. Legs: yellowish without marks or pattern. Wings hyaline except costal and subcostal area apically white, with brown venation. Abdomen: tergites I to X light brown without mark or pattern. Sternites I and II light brown; sternites III to VII uniformly ecru without marks or pattern; sternites VIII and IX light brown. Cerci ecru. Genitalia (Fig. 46) ecru except inner margin of segment I medium brown. Forewing (Fig. 45): pterostigma with approx. four cross-veins not reaching subcostal vein; double intercalary veins shorter to almost equal to distance between corresponding main veins. Hindwing similar to Fig. 17 except two longitudinal veins reaching margin. Genitalia (Fig. 46): basal segment with inner margin not expanded apically; segment I and II almost completely fused; inner margin at the apex of segment I and base of segment II with a triangular well-marked expansion; segment III almost globular.

Distribution and habitat. This species was collected in three different wadis at altitudes between $200 \mathrm{~m}$ and $750 \mathrm{~m}$. Larvae occur in small streams, generally very shallow (a few centimeters to $20 \mathrm{~cm}$ ) with moderate current. The substrate was a mix of sand, cobbles and rocks (Figs 50, 51, 52). This species was sympatric with the two other species of Labiobaetis and C. cf. soldani, but generally less abundant than other species. 
Table 2. Estimates of evolutionary divergence between major haplogroups of Labiobaetis species (using corrected $\mathrm{p}$ distances). In brackets are indicated the minimum and maximum distances.

\begin{tabular}{|c|c|c|c|c|c|}
\hline & $\begin{array}{l}\text { Labiobaetis } \\
\text { glaucus }\end{array}$ & $\begin{array}{l}\text { Labiobaetis } \\
\text { glaucus }\end{array}$ & $\begin{array}{l}\text { Labiobaetis } \\
\text { glaucus }\end{array}$ & $\begin{array}{l}\text { Labiobaetis } \\
\text { alahmadii }\end{array}$ & $\begin{array}{l}\text { Labiobaetis } \\
\text { potamoticus }\end{array}$ \\
\hline $\begin{array}{l}\text { Labiobaetis } \\
\text { glaucus }\end{array}$ & $\begin{array}{c}0 \\
(0.0-0.004) \\
\end{array}$ & & & & \\
\hline $\begin{array}{l}\text { Labiobaetis } \\
\text { glaucus }\end{array}$ & $\begin{array}{c}0.02 \\
(0.06-0.021) \\
\end{array}$ & $\begin{array}{c}0 \\
(0.0-0.012) \\
\end{array}$ & & & \\
\hline $\begin{array}{l}\text { Labiobaetis } \\
\text { glaucus }\end{array}$ & $\begin{array}{c}0.023 \\
(0.021-0.025) \\
\end{array}$ & $\begin{array}{c}0.012 \\
(0.012)\end{array}$ & 0 & & \\
\hline $\begin{array}{l}\text { Labiobaetis } \\
\text { alahmadii }\end{array}$ & $\begin{array}{c}0.196 \\
(0.194-0.199) \\
\end{array}$ & $\begin{array}{c}0.173 \\
(0.173) \\
\end{array}$ & $\begin{array}{c}0.183 \\
(0.183) \\
\end{array}$ & 0 & \\
\hline $\begin{array}{l}\text { Labiobaetis } \\
\text { potamoticus }\end{array}$ & $\begin{array}{c}0.179 \\
(0.173-0.183) \\
\end{array}$ & $\begin{array}{c}0.182 \\
(0173-0.189) \\
\end{array}$ & $\begin{array}{c}0.168 \\
(0.168) \\
\end{array}$ & $\begin{array}{c}0.168 \\
0.162-0.173 \\
\end{array}$ & $\begin{array}{c}0.003 \\
(0.00-0.008)\end{array}$ \\
\hline Labiobaetis spp. & $(0.178-0.254)$ & $(0.178-0.250)$ & $(0.173-0.255)$ & $(0.153-0.293)$ & $(0.042-0.256)$ \\
\hline
\end{tabular}

\section{Molecular results}

The mitochondrial reconstruction clearly recovers $L$. potamoticus and $L$. alahmadii as monophyletic clades (BS (Bootstrap support) of $83 \%$ and $100 \%$ respectively), with intraspecific K2P distances below 1\% (Table 2). Labiobaetis glaucus is also highly supported as a monophyletic clade (BS of 100\%), with the three populations (KSA, Mayotte, and South Africa) supported as monophyletic sister-clades (BS of $87 \%, 81 \%$ and $90 \%$ respectively). The sister group of Labiobaetis potamoticus is an undescribed species from South Africa; the distance between the two taxa is slightly higher than intraspecific distance (between 4.2 and 5.1\%). Labiobaetis potamoticus possesses high distances to all the other species included in the study (16.2 to $25.5 \%$ ). The relationships of $L$. alahmadii and L. glaucus with other species of Afrotropical and Palaearctic origins also are unclear and have no molecular support (Fig. 11). Both species are highly distant from any other taxa (Table 2).

\section{Discussion}

Labiobaetis alahmadii is morphologically similar to L. glaucus as well as with the Palaearctic species L. cleopatrae (Thomas \& Soldán, 1989) and L. balcanicus and the Afrotropical species L. boussoulius (Gillies, 1993). The shape of the distolateral process of the second segment of the labial palp is of taxonomic importance to separate the different species: more elongated and curved in L. potamoticus (Fig. 8) and L. balcanicus whereas shorter and more rounded in L. glaucus (Fig. 39), L. cleopatrae, and L. boussoulius. L. alahmadii differs from these four species by the presence of short spatulate setae on the dorsal margin of the tibia and tarsi (Fig. 26) (Gillies 1993; Lugo-Ortiz et al. 2000; Müller-Liebenau and Soldán 1981; Thomas and Soldán 1989).

Labiobaetis glaucus is rather similar to L. cleopatrae. The two species differ by minor characters such as: the shape of the margin between prostheca and mola of the right and 
left mandibles (straight in L. glaucus (Fig. 35) and curved in L. cleopatrae), the shape of the apex of the segment II of the maxillary palp (simple in L. glaucus (Fig. 38) and slightly curved with a small apical protuberance in L. cleopatrae), distal margin of tergites (triangular and pointed simple spines in L. glaucus (Fig. 43) and double triangular slightly worn spines in L. cleopatrae), tarsal claw less curved and less stout in L. glaucus (Fig. 40) than in L. cleopatrae. These statements are based on the comparison of material of $L$. glaucus stored in the collection of the MZL with the original description of L. cleopatrae. According to the variability already noticed in L. glaucus and the known synonyms of the species (Lugo-Ortiz et al. 2000; Lugo-Ortiz and McCafferty 1997), we cannot exclude that $L$. cleopatrae is a possible junior synonym of $L$. glaucus.

Labiobaetis potamoticus and L. piscis Lugo-Ortiz \& McCafferty, 1997 share several important synapomorphies: shape of the distomedial projection of segment II of the labial palp (Fig. 8); spines of the paraprocts (Fig. 15) and setation of the inner margin of the femur (Lugo-Ortiz and McCafferty 1997). These two species differ primarily by the shape of segment III of the labial palp (more slender and with the inner margin more concave in L. piscis) and the setation of the ventral margin of femora (villopore reduced but always present in L. potamoticus, absent in L. piscis; setae almost as long in ventral margin than dorsal margin in L. piscis, much shorter in L. potamoticus). Labiobaetis potamoticus and $L$. piscis show a peculiar pattern on the abdominal tergites (Fig. 19). A similar pattern is also present in L. tripunctatus Gillies, 1994 and L. punctatus Gattolliat, 2001 (Gattolliat 2001; Gillies 1994). Labiobaetis tripunctatus has no hindwings and only six pairs of gills (Gillies 1994), while L. punctatus is strictly endemic to Madagascar and clearly differs by the excavation of the maxillary palp segment II, the spination of the distal margin of tergites and the setation of the labrum (Gattolliat 2001). Genetically, L. potamoticus does not appear related to either L. piscis or L. punctatus. No sequences are available for L. tripunctatus. The sister group of Labiobaetis potamoticus is an undescribed species from South Africa. The two species are morphologically similar but also exhibit some differences especially in the femoral setation. This tends to confirm that the two sister taxa are closely related species but are not conspecific. The status of this undescribed species will be discussed in a revision of Labiobaetis from the Afrotropical Region (Kaltenbach, comm. pers.). Labiobaetis potamoticus was initially identified as L. balcanicus (Thomas and Sartori 1989). The two species share important characters (paraproct, antenna, labrum, mandibles, abdominal pattern), but also clearly differ by the shape of the spines of the distal margin of abdominal tergites (apically pointed in L. potamoticus (Fig. 14) whereas apically rounded in L. balcanicus) and the shape of the distomedial projection of the segment II of the labial palp (shorter and more slender in L. potamoticus (Fig. 8)).

The imagos of the different species of Labiobaetis are generally similar. The presence or absence of the hindwings and the shape of the genital plates are the main characters to separate the species (Gattolliat 2001). The male imago of Labiobaetis potamoticus cannot be separated from most other species of Labiobaetis with hindwings and broad, apically flat genital plates. Labiobaetis glaucus and L. boussoulius differ from most other species of the genus by the presence of a well-marked triangular expansion on the inner margin of the gonopods (Fig. 3 in Gillies 1993). A similar triangular expansion on 


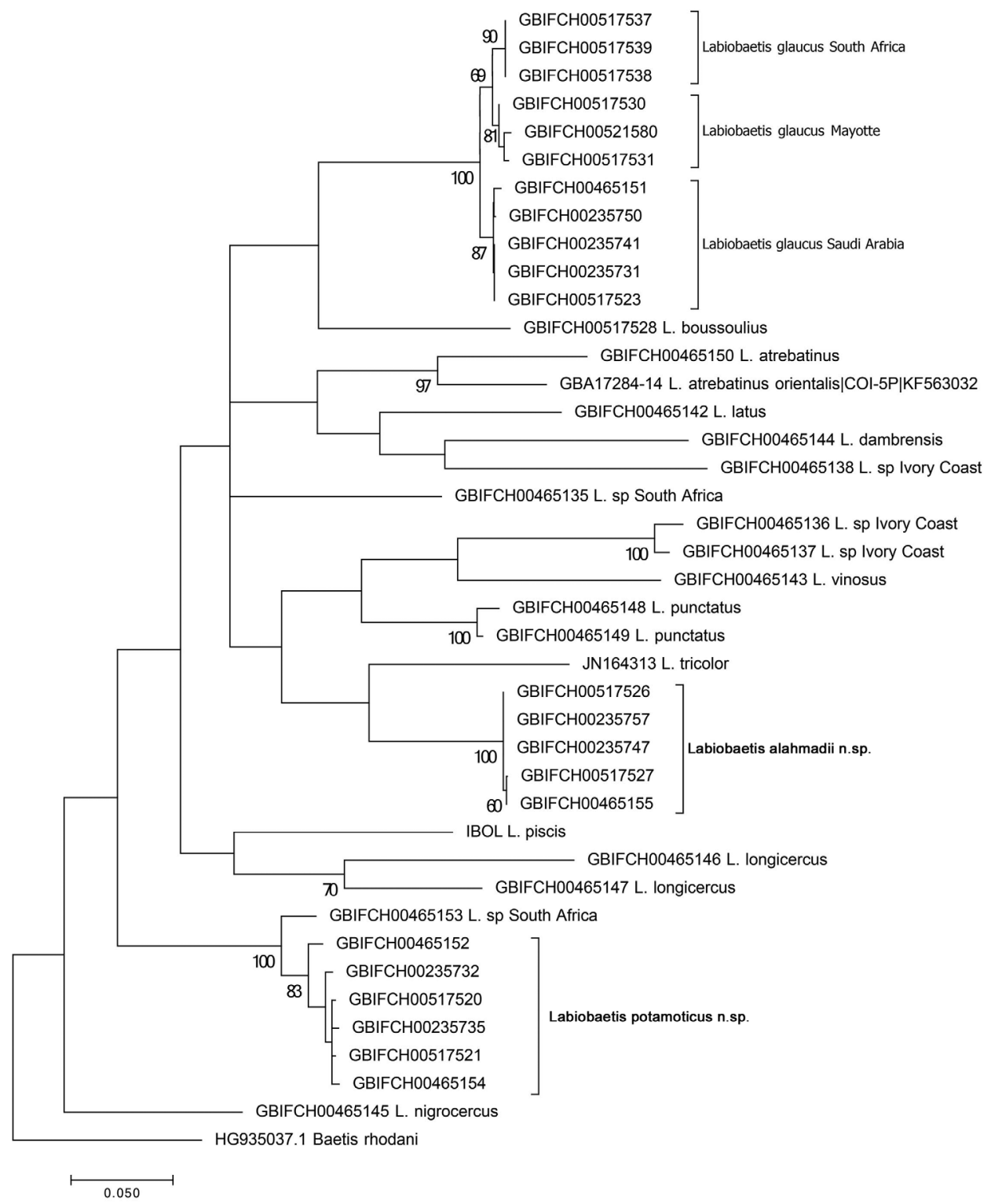

Figure 53. Maximum Likelihood (ML) consensus tree reconstructed for 40 haplotypes of Labiobaetis spp. Tree drawn to scale, branch lengths measured in number of substitutions per site, deeper nodes labelled above branches with Maximum Likelihood bootstrap support (>50\%).

the inner margin of the gonopods is present, but even more pronounced, in L. tricolor Tshernova, 1928 (fig. 111a in Müller-Liebenau 1969).

The discovery of $L$. glaucus in KSA is rather unexpected despite that this species is widely distributed in Afrotropical Region (South Africa, Angola, Kenya, Lesotho, Namibia, and Zimbabwe (de Moor et al. 2000; Lugo-Ortiz et al. 2000); Comoros islands 
(N. Mary unpublished data)). We examined specimens of populations from South Africa, Mayotte (Comoros Islands) and KSA. We found no clear morphological differences between them and they fully correspond to the original description and subsequent redescriptions (Agnew 1961; Lugo-Ortiz and McCafferty 1997; Lugo-Ortiz et al. 2000). As already mentioned (Lugo-Ortiz et al. 2000), segment II of the labial palp may have a more or less developed distomedial projection and the abdominal pattern may differ between populations. Genetically, populations from South Africa, Mayotte and KSA form a highly supported monophyletic clade. Genetic distances between the three populations are clearly of intraspecific range especially if we consider that intermediate populations from East and North-East Africa are not included in the analysis.

\section{Key to the larvae of Labiobaetis known from KSA}

$1 \quad$ Ventral margin of femora with abundant pointed setae (Fig. 10); labial palp with a moderately developed, relatively slender distolateral process (Fig. 8); margin of paraproct with abundant regular spines (Fig. 15); abdominal segments I-X brown with six ecru dots (Fig. 19)

Labiobaetis potamoticus sp. $\mathbf{n}$.

- Ventral margin of femora with scarce short setae (Figs 25, 40); labial palp with a well-developed distolateral process (Figs 24, 39); margin of paraproct with 8-10 spines increasing in length (Figs 31, 44); abdominal segments I-X with different patterns 2

2 Dorsal margin of tibiae and tarsi with a complete row of spatulate setae (Fig. 26); mesothorax medium brown with a double V-shaped yellow pattern; tergites I-VIII medium brown with two broad ecru spot; tergites IX and X yellow (Figs 32, 33)

Labiobaetis alabmadii sp. $\mathbf{n}$.

- $\quad$ Dorsal margin of tibiae and tarsi with scattered minute pointed setae; mesothorax (Fig. 40) uniformly brown (Fig. 47); tergites II-IV and VII-VIII medium brown with small ecru spot, other tergites yellow

Labiobaetis glaucus

\section{Acknowledgements}

We want to thank Marion Podolak for providing much assistance with the molecular analyses and to Michel Sartori for image processing. Many thanks to Drs. Mostafa Sharaf, Mahmoud Saleh Seleem, Hassan Fadl (all KSU), and Christian Monnerat (CSCF, Switzerland) for their help and friendship during the fieldtrip in 2012. We express our gratitude to Pavel Sroka and Ina Ferreira for reviewing the manuscript. This work was supported in part by the Deanship of Scientific Research at King Saud University, Riyadh, KSA. Special thanks to other team members of the King Saud University Museum of Arthropods, College of Food and Agriculture Sciences, Department of Plant Protection, King Saud University for assistance during this study. 


\section{References}

Agnew JD (1961) New Baetidae (Ephem.) from South Africa. Novos Taxa Entomologicos 25: 3-18. Al-Ghamdi HS, Abu-Zinadah OA (1998) Study of freshwater fish of the Mid-Western Region of Saudi Arabia. Journal of King Abdulaziz University-Science 10: 39-44. https://doi. org/10.4197/Sci.10-1.4

Alkahem AF, Behnke RJ (1983) Freshwater fishes of Saudi Arabia. Fauna of Saudi Arabia 5: $545-567$.

Alkolibi FM (2002) Possible effects of global warming on agriculture and water resources in Saudi Arabia: Impacts and responses. Climatic Change 54: 225-245. https://doi. org/10.1023/A:1015777403153

Alshareef AS (1995) Geography of Saudi Arabia. Part 1. Al-Mureekh Publication, Riyadh, Saudi Arabia.

Al-Rashed MF, Sherif MM (2000) Water resources in the GCC countries: An overview. Water Resources Management 14: 59-75. https://doi.org/10.1023/A:1008127027743

Ball SL, Hebert PDN, Burian SK, Webb JM (2005) Biological identifications of mayflies (Ephemeroptera) using DNA barcodes. Journal of the North American Benthological Society 24: 508-524. https://doi.org/10.1899/04-142.1

Crosskey RW, Büttiker W (1982) Insects of Saudi Arabia. Diptera: Fam. Simuliidae. Fauna of Saudi Arabia 4: 398-446.

de Moor FC, Barber-James HM, Harrison AD, Lugo-Ortiz CR (2000) The macroinvertebrates of the Cunene river from the Ruacana falls to the river mouth and assessment of the conservation status of the river. African Journal of Aquatic Science 25: 105-122. https:// doi.org/10.2989/160859100780177857

Edgell HS (2006) Arabian deserts: Nature, origin and evolution. Springer, Dordrecht, The Netherlands, 592 pp. https://doi.org/10.1007/1-4020-3970-0

Folmer O, Black M, Hoeh W, Lutz R, Vrijenhoek R (1994) DNA primers for amplification of mitochondrial cytochrome c oxidase subunit I from diverse metazoan invertebrates. Molecular Marine Biology and Biotechnology 3: 294-299.

Fujitani T (2008) The family Baetidae from Japan. In: Hauer FR, Stanford JA, Newell RL (Eds) International Advances in the Ecology, Zoogeography and Systematics of Mayflies and Stoneflies. University of California Press, Berkeley, 205-218. https://doi.org/10.1525/ california/9780520098688.003.0015

Fujitani T, Hirowatari T, Tanida K (2003) Genera and species of Baetidae in Japan: Nigrobaetis, Alainites, Labiobaetis, and Tenuibaetis n. stat. (Ephemeroptera). Limnology 4: 121-129. https://doi.org/10.1007/s10201-003-0105-2

Gattolliat J-L (2001) Six new species of Labiobaetis Novikova \& Kluge (Ephemeroptera: Baetidae) from Madagascar with comments on the validity of the genus. Annales de Limnologie 37: 97-123. https://doi.org/10.1051/limn/2001013

Gattolliat J-L, Monaghan MT, Sartori M, Elouard JM, Barber-James H, Derleth P, Glaizot O, de Moor F, Vogler AP (2008) A molecular analysis of the Afrotropical Baetidae. In: Hauer FR, Stanford JA, Newell RL (Eds) International Advances in the Ecology, Zoogeography and Systematics of Mayflies and Stoneflies. University of California Press, Berkeley, 219-232. https://doi.org/10.1525/california/9780520098688.003.0016 
Gattolliat J-L, Nieto C (2009) The family Baetidae (Insecta: Ephemeroptera): synthesis and future challenges. Aquatic Insects 31: 41-62. https://doi.org/10.1080/01650420902812214 Gattolliat J-L, Sartori M (2008) Order Ephemeroptera. In: van Harten A (Ed) Arthropod Fauna of the UAE. Dar Al Ummah, Abu Dhabi, 47-83.

Gattolliat J-L, Staniczek A (2011) New larvae of Baetidae (Insecta: Ephemeroptera) from Espiritu Santo, Vanuatu. Stuttgarter Beiträge zur Naturkunde A, Neue Serie 4: 75-82

Gattolliat J-L, Vuataz L, Sartori M (2012) First contribution to the mayflies of Jordan. Zoology in the Middle East 56: 91-110. https://doi.org/10.1080/09397140.2012.10648945

Gattolliat JL, Cavallo E, Vuataz L, Sartori M (2015) DNA barcoding of Corsican mayflies (Ephemeroptera) with implications on biogeography, systematics and biodiversity. Arthropod Systematics \& Phylogeny 73: 3-18.

Gillies MT (1993) Descriptions of some Afrotropical Baetidae (Ephemeroptera) II. Baetis Leach, s.l., West African species. Aquatic Insects 15: 213-223. https://doi. org/10.1080/01650429309361522

Gillies MT (1994) Descriptions of some Afrotropical Baetidae (Ephemeroptera) II. Baetis Leach, s.l., East African species. Aquatic Insects 16: 105-118. https://doi. org/10.1080/01650429409361543

Kingdom of Saudi Arabia Ministry of Foreign Affairs (2015) Dams in the Kingdom. http:// www.mofa.gov.sa/sites/mofaen/ServicesAndInformation/news/GovernmentNews/Pages/ NewsArticleID72349.aspx [accessed November 2015]

Kluge NJ (2012) [Status of the mayfly species described by O.A. Tshernova from Amur Nasin in the genus Baetis Leach (Ephemeroptera, Baetidae)]. Entomologicheskoe Obozrenie 91: 154-156. [in Russian]

Kluge NJ, Novikova EA (2016) New tribe Labiobaetini tribus n., redefinition of Pseudopannota Waltz \& McCafferty 1987 and descriptions of new and little known species from Zambia and Uganda. Zootaxa 4169: 1-43. https://doi.org/10.11646/zootaxa.4169.1.1

Kubendran T, Balasubramanian C, Selvakumar C, Gattolliat JL, Sivaramakrishnan KG (2015) Contribution to the knowledge of Tenuibaetis Kang \& Yang 1994, Nigrobaetis Novikova \& Kluge 1987 and Labiobaetis Novikova \& Kluge 1987 (Ephemeroptera: Baetidae) from the Western Ghats (India). Zootaxa 3957: 188-200. https://doi.org/10.11646/zootaxa.3957.2.3

Kumar S, Stecher G, Tamura K (2016) MEGA7: Molecular Evolutionary Genetics Analysis version 7.0 for bigger datasets. Molecular Biology and Evolution 33: 1870-1874. https:// doi.org/10.1093/molbev/msw054

Lugo-Ortiz CR, de Moor FC, Barber-James HM (2000) A taxonomic and ecological review of Pseudocloeon glaucum (Agnew) (Ephemeroptera : Baetidae). African Entomology 8: 281-288. Lugo-Ortiz CR, McCafferty WP (1997) Labiobaetis Novikova \& Kluge (Ephemeroptera: Baetidae) from the Afrotropical region. African Entomology 5: 241-260.

Lugo-Ortiz CR, McCafferty WP, Waltz RD (1999) Definition and reorganization of the genus Pseudocloeon (Ephemeroptera: Baetidae) with new species descriptions and combinations. Transactions of the American Entomological Society 125: 1-37.

McCafferty WP, Waltz RD (1995) Labiobaetis (Ephemeroptera: Baetidae): new status, new North American species, and related new genus. Entomological News 106: 19-28.

Monaghan MT, Gattolliat JL, Sartori M, Elouard JM, James H, Derleth P, Glaizot O, de Moor F, Vogler AP (2005) Trans-oceanic and endemic origins of the small minnow mayflies 
(Ephemeroptera, Baetidae) of Madagascar. Proceedings of The Royal Society B-Biological Sciences 272: 1829-1836. https://doi.org/10.1098/rspb.2005.3139

Müller-Liebenau I (1969) Revision der europäischen Arten der Gattung Baetis Leach, 1815 (Insecta, Ephemeroptera). Gewässer und Abwässer 48/49: 1-214.

Müller-Liebenau I, Soldán T (1981) Baetis balcanicus sp. n., a new species of the genus Baetis from Bulgaria and Greece. Spixiana 4: 291-295.

Rutschmann S, Detering H, Simon S, Funk DH, Gattolliat J-L, Hughes SJ, Raposeiro PM, DeSalle R, Sartori M, Monaghan MT (2017) Colonization and diversification of aquatic insects on three Macaronesian archipelagos using 59 nuclear loci derived from a draft genome. Molecular Phylogenetics and Evolution 107: 27-38. https://doi.org/10.1016/j. ympev.2016.10.007

Rutschmann S, Gattolliat JL, Hughes SJ, Baez M, Sartori M, Monaghan MT (2014) Evolution and island endemism of morphologically cryptic Baetis and Cloeon species (Ephemeroptera, Baetidae) on the Canary Islands and Madeira. Freshwater Biology 59: 2516-2527. https://doi.org/10.1111/fwb.12450

Salles FF, Gattolliat J-L, Angeli KB, De-Souza MR, Goncalves IC, Nessimian JL, Sartori M (2014) Discovery of an alien species of mayfly in South America (Ephemeroptera). ZooKeys 399: 1-16. https://doi.org/10.3897/zookeys.399.6680

Sartori M (1991) The Mayfly fauna (Insecta; Ephemeroptera) of the Arabian Peninsula (Part 3). Fauna of Saudi Arabia 12: 242-245.

Sartori M, Gillies MT (1990) Further records of mayflies (Insecta: Ephemeroptera) from the Arabian Peninsula. Leptophlebiidae and Baetidae. Fauna of Saudi Arabia 11: 10-17.

Sorman AU, Abdulrazzak MJ (1993) Infiltration-recharge through wadi beds in arid regions. Hydrological Sciences Journal 38: 173-186. https://doi.org/10.1080/02626669309492661

Tamura K, Nei M (1993) Estimation of the number of nucleotide substitutions in the control region of mitochondrial DNA in humans and chimpanzees. Molecular Biology and Evolution 10: 512-526.

Thomas AGB, Sartori M (1989) Mayflies (Insecta, Ephemeroptera) of Saudi Arabia. Fauna of Saudi Arabia 10: 87-94. https://doi.org/10.1051/limn/1989006

Thomas AGB, Soldán T (1989) Baetis cleopatrae n. sp., Ephéméroptère nouveau d'Egypte (Baetidae). Annales de Limnologie 25: 55-59.

Vuataz L, Sartori M, Wagner A, Monaghan MT (2011) Toward a DNA Taxonomy of Alpine Rhithrogena (Ephemeroptera: Heptageniidae) Using a Mixed Yule-Coalescent Analysis of Mitochondrial and Nuclear DNA. Plos One 6: e19728. https://doi.org/10.1371/journal. pone.0019728

Webb JM, Jacobus LM, Funk DH, Zhou X, Kondratieff B, Geraci CJ, DeWalt RE, Baird DJ, Richard B, Phillips I, Hebert PDN (2012) A DNA Barcode Library for North American Ephemeroptera: Progress and Prospects. Plos One 7. https://doi.org/10.1371/journal. pone.0038063

Whitton BA, Khoga TM, Arif IA (1986) Water chemistry and algal vegetation of streams on the Asir Mountains, Saudi Arabia. Hydrobiologia 133: 97-106. https://doi.org/10.1007/ BF00031859

Wuillot J, Gillies MT (1993) Cheleocloeon, a new genus of Baetidae (Ephemeroptera) from West Africa. Revue d'Hydrobiologie tropicale 26: 213-217. 